\title{
Covered Stents versus Uncovered Stents for Unresectable Malignant Biliary Strictures: A Meta-Analysis
}

\author{
Ming-Yu Chen, ${ }^{1,2}$ Jia-Wei Lin, ${ }^{2}$ He-Pan Zhu, ${ }^{2}$ Bin Zhang, ${ }^{2}$ Guang-Yi Jiang, \\ Pei-Jian Yan, ${ }^{2}$ and Xiu-Jun Cai ${ }^{1,2}$ \\ ${ }^{1}$ Department of General Surgery, Sir Run Run Shaw Hospital, College of Medicine, Zhejiang University, Hangzhou, Zhejiang 310016, \\ China \\ ${ }^{2}$ Zhejiang University, Zhejiang 310020, China
}

Correspondence should be addressed to Xiu-Jun Cai; caixiujunzju@163.com

Received 15 December 2015; Revised 29 January 2016; Accepted 17 February 2016

Academic Editor: Michel Kahaleh

Copyright (c) 2016 Ming-Yu Chen et al. This is an open access article distributed under the Creative Commons Attribution License, which permits unrestricted use, distribution, and reproduction in any medium, provided the original work is properly cited.

\begin{abstract}
Aim. To summarize the covered or uncovered SEMS for treatment of unresectable malignant distal biliary obstruction, comparing the stent patency, patient survival, and incidence of adverse events between the two SEMSs. Methods. The meta-analysis search was performed independently by two of the authors, using MEDLINE, EMBASE, OVID, and Cochrane databases on all studies between 2010 and 2015. Pooled effect was calculated using either the fixed or the random effects model. Results. Statistics shows that there is no difference between SEMSs in the hazard ratio for patient survival (HR 1.04; 95\% CI, 0.92-1.17; $P=0.55$ ) and stent patency (HR 0.87, 95\% CI: 0.58 to $1.30, P=0.5$ ). However, incidence of adverse events (OR: $0.74,95 \%$ CI: 0.57 to $0.97, P=0.03$ ) showed significant different results in the covered SEMS, with dysfunctions events (OR: 0.75, 95\% CI: 0.56 to $1.00, P=0.05)$ playing a more important role than complications (OR: $0.87,95 \%$ CI: 0.58 to $1.30, P=0.50$ ). Conclusions. Covered SEMS group had lower incidence of adverse events. There is no significant difference in dysfunctions, but covered SEMS trends to be better, with no difference in stent patency, patient survival, and complications.
\end{abstract}

\section{Introduction}

Stenting has become widely accepted as the treatment of unresectable distal malignant biliary obstructions (UDMBO), since Soehendra and Reynders-Frederix [1] first introduced transpapillary biliary drainage in 1980 . However, the patency and incidence of adverse events (dysfunctions and complications) have an influence on the quality of life. Stent dysfunction comprised stent migration, stent occlusion causing tumor over- and/or ingrowth, which included proximal overgrowth, distal overgrowth, proximal and distal overgrowth, ingrowth, and encrustation (sludge) [2]. It can be confirmed by subsequent radiologic studies, including computed tomography, percutaneous cholangiography, and endoscopic retrograde cholangiopancreatography, and based on lab test such as recurrent jaundice. Complication comprised hemorrhage, cholecystitis, pancreatitis retroperitoneal, perforation, and cholangitis (medical therapy), which was suspected based on clinical symptoms and signs. There are various types of stents used for the management of UDMBO: plastic stents, self-expandable metallic stent (SEMS) such as uncovered and covered stents, and recent developed bioabsorbable or biodegradable stents [3]. SEMS is considered to be the most cost-effective biliary stent for treatment of unresectable malignant biliary obstructions (UDMBO), but there is still some debate on the selection of covered or uncovered SEMS in distal duct strictures. The main disadvantage of covered SEMS is migration, because of nonembedded stent body, while the uncovered SEMSs which were embedded into the biliary wall are resistant to migration, because of their mesh structure and selfexpandability. Unluckily, the tumor ingrowth (TI) via the stent mesh is the main cause of occlusion in uncovered SEMS. Although there are some randomized studies comparing covered and uncovered SEMS, the results are different according to each study. One [4] showed that covered SEMS had longer patency than uncovered SEMS; another $[2,5]$ revealed no statistically significant difference. Interestingly, 
the latest meta-analysis revealed that covered SEMS did not appear to have longer patency, and benefits brought by the two SEMSs to patients were not clear [6]. Unfortunately, the meta-analysis studies which focused on complications did not pay attention to the difference in patient survival and incidence of adverse event and dysfunction events. Therefore, it is essentially necessary to get a comprehensive understanding on the difference between uncovered and covered SEMS for the treatment of unresectable distal malignant biliary obstructions, especially stent patency, patient survival, and incidence of adverse events.

\section{Materials and Methods}

This meta-analysis adhered to the guidelines of Preferred Reporting Items for Systematic Reviews and Meta-Analyses (PRISMA).

2.1. Study Selection. The meta-analysis search was performed independently by two of the authors (Mr. Jiang and Mr. Yan), using MEDLINE, EMBASE, OVID, and Cochrane databases. The search was performed on all studies between 2010 and 2015 to compare covered and uncovered SEMS for unresectable distal malignant biliary obstructions. The search strategy was based on the following Medical Subject Heading terms (MeSH): "unresectable," "malignant biliary obstructions," "distal biliary obstructions," "distal biliary strictures," "covered," "uncovered," "self-expandable metallic stents," and "SEMS." Only studies on humans and in English and Chinese language were considered for inclusion. Reference lists of all retrieved articles were manually searched for additional studies.

2.2. Data Extraction and Conversion. Data extraction was performed independently by two reviewers (Mr. Jiang and Mr. Yan, resp.). The following parameters for each study included (1) first author, the year of publication, and the study type; (2) the number and characteristics of patients; (3) the outcome of the trials including number or incidence of adverse events (dysfunction and complication) and HR of elevated stent patency and patient survival, as well as their 95\%. If available, the HRs with their 95\% CIs and $P$ values were collected from the original article or the corresponding E-mails. If not, we calculated HRs and their $95 \%$ confidence interval using the data of observed deaths, the data of samples in each group, or the data provided by the authors. If only Kaplan-Meier curves were available, we extracted data from the graphical survival plots and estimated the HRs. All the calculations mentioned above were based on the methods provided by Tierney et al. [14] and Parmar et al. [15].

2.3. Inclusion Criteria. The studies included in the metaanalysis had to fulfill the following criteria: (1) they should compare the original outcomes of covered and uncovered SEMSs for the treatment of unresectable distal malignant biliary obstructions; (2) they should report on at least stent patency, patient survival, and incidence of adverse events; (3) if dual (or multiple) studies were reported by the same

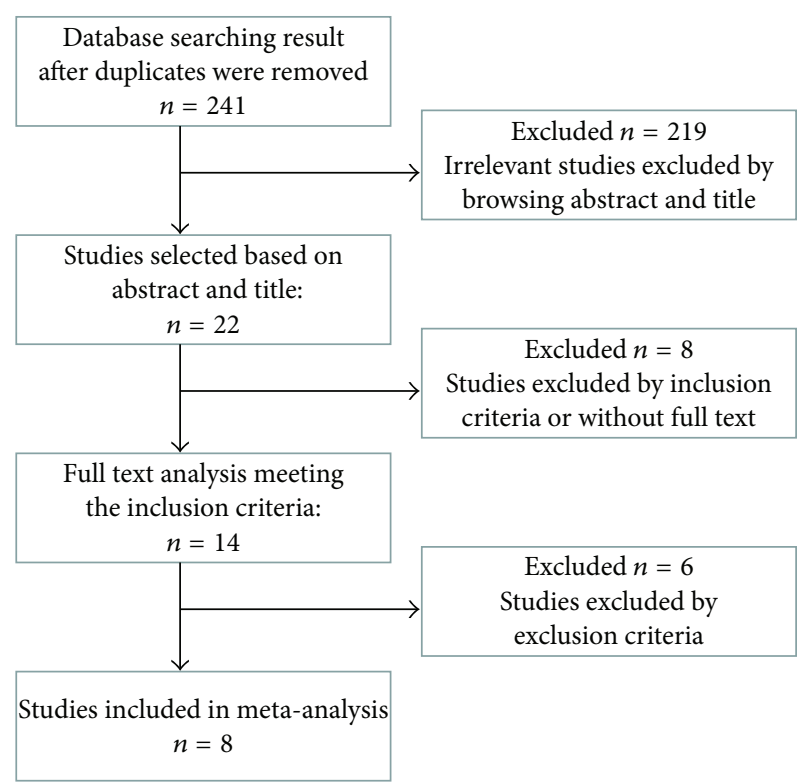

FIGURE 1: Flowchart showing selection of studies for meta-analysis.

institution and/or authors, only the most recent publication or the highest quality of studies was included.

2.4. Exclusion Criteria. The following studies were excluded: (1) those dealing with biliary malignant strictures or obstructions with second stents; (2) those using other types of stents, such as plastic stents; (3) those with no clearly reported outcomes; and (4) abstracts, letters, editorials and expert opinions, reviews without original data, case reports, and studies lacking control groups.

2.5. Statistical Analysis. The meta-analysis was performed using Review Manager (RevMan), version 5.3. Stent patency and patient survival were analyzed using estimation of hazard ratios (HRs) with a 95\% confidence interval (95\% CI). Pooled effect was calculated using either the fixed or the random effects model. The test of heterogeneity of combined HRs was carried out using Cochran's $Q$ test and Higgins $I$ squared statistic. If the $I^{2}$ statistic was $>50 \%$, we considered heterogeneity to be present. If the probability of a chance occurrence was less than $5 \%(P<0.05)$, all statistical data were considered significant.

\section{Results}

3.1. Selection of Trials. After initial screening, of the 14 clinical trials $[2,5,7-13,16-20]$ which initially met the inclusion criteria, $2[17,20]$ did not display the specific comparison of the effects of covered and uncovered SEMS, 1 [16] used second stents, and 1 [5] dealt with patients who had received or were receiving chemotherapy, and $2[18,19]$ did not provide enough original data. Finally, 8 studies, including 2 retrospective studies and 6 prospective randomized studies, matched the selection criteria and were published between 2010 and 2015 (Figure 1). The characteristics of these 8 studies are summarized in Table 1 . The 8 studies included a total of 


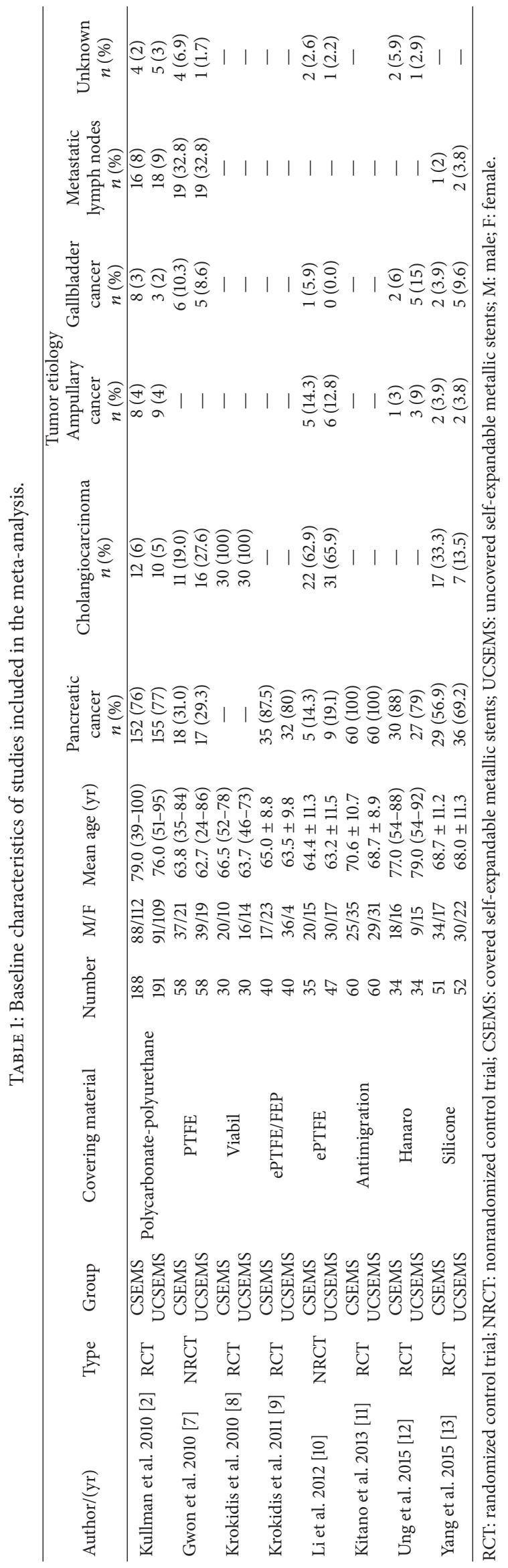




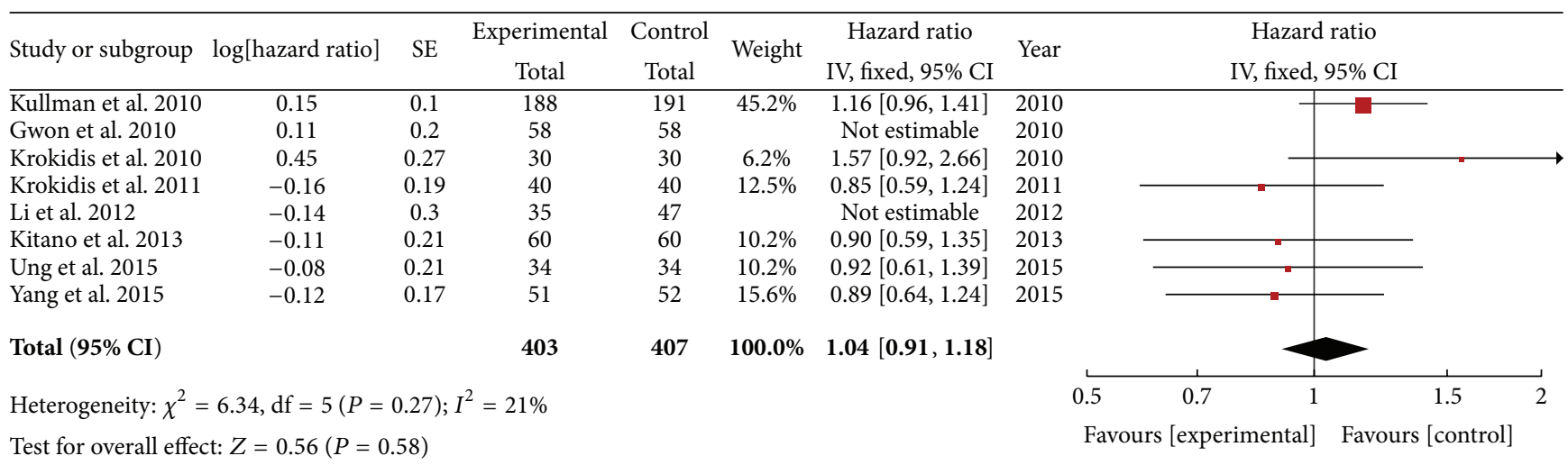

FIGURE 2: Forest plot of studies evaluating hazard ratios of patient survival.

\begin{tabular}{|c|c|c|c|c|c|c|c|c|c|c|}
\hline Study or subgroup & $\log [$ hazard ratio $]$ & SE & $\begin{array}{c}\text { Covered SEMS } \\
\text { Total }\end{array}$ & $\begin{array}{l}\text { Uncovered SEMS } \\
\text { Total }\end{array}$ & Weight & $\begin{array}{l}\text { Hazard ratio } \\
\text { IV, fixed, 95\% CI }\end{array}$ & \multicolumn{4}{|c|}{$\begin{array}{c}\text { Hazard ratio } \\
\text { IV, fixed, 95\% CI }\end{array}$} \\
\hline Krokidis et al. 2010 & 1.21 & 0.7 & 30 & 30 & $1.7 \%$ & $3.35[0.85,13.22]$ & & & & \\
\hline Krokidis et al. 2011 & -1.32 & 0.69 & 40 & 40 & $1.8 \%$ & $0.27[0.07,1.03]$ & & & & \\
\hline Gwon et al. 2010 & -0.35 & 0.4 & 58 & 58 & $5.3 \%$ & $0.70[0.32,1.54]$ & & & & \\
\hline Li et al. 2012 & -0.35 & 0.32 & 35 & 47 & $8.4 \%$ & $0.70[0.38,1.32]$ & & & & \\
\hline Yang et al. 2015 & -0.17 & 0.27 & 51 & 52 & $11.7 \%$ & $0.84[0.50,1.43]$ & & & & \\
\hline Kitano et al. 2013 & -0.43 & 0.23 & 60 & 60 & $16.2 \%$ & $0.65[0.41,1.02]$ & & & & \\
\hline Ung et al. 2015 & -0.05 & 0.2 & 34 & 34 & $21.4 \%$ & $0.95[0.64,1.41]$ & & - & & \\
\hline Kullman et al. 2010 & 0.18 & 0.16 & 188 & 191 & $33.4 \%$ & $1.20[0.87,1.64]$ & & & & \\
\hline Total $(95 \%$ CI $)$ & & & 496 & 512 & $100.0 \%$ & $0.91[0.76,1.09]$ & & & & \\
\hline \multicolumn{7}{|c|}{ Heterogeneity: $\chi^{2}=12.87, \mathrm{df}=7(P=0.08) ; I^{2}=46 \%$} & 0.5 & 0.7 & 1.5 & 2 \\
\hline \multicolumn{7}{|c|}{ Test for overall effect: $Z=0.98(P=0.33)$} & & $\begin{array}{l}\text { Covered } \\
\text { SEMS }\end{array}$ & $\begin{array}{l}\text { Uncovered } \\
\text { SEMS }\end{array}$ & \\
\hline
\end{tabular}

FIGURE 3: Forest plot of studies evaluating hazard ratios of stent patency.

1067 patients: 524 in the covered SEMS group and 533 in the uncovered SEMS group. The sample size of each study varied from 30 to 191 patients. The proportion of men (OR $=1.02,95 \% \mathrm{CI}: 0.80-1.31, P=0.85)$ and the proportion of pancreatic cancer in tumor etiology $(\mathrm{OR}=0.97,95 \% \mathrm{CI}: 0.69-$ $1.36, P=0.87)$ were not significant.

3.2. Patient Survival. All 8 studies reported patient survival. No statistical difference existed between the covered SEMS and uncovered SEMS in the hazard ratio of patient survival (HR 1.04; 95\% CI, 0.92-1.17; $P=0.55$ ). There is no heterogeneity among the 8 studies, and a fixed-effect model was used (Figure 2).

3.3. Stent Patency. As a fixed-effect model was used, the meta-analysis from the 8 studies also showed no significant difference of stent patency between two groups (HR 0.87, 95\% CI: 0.58 to $1.30, P=0.5$ ) (Figure 3 ).

3.4. Complications and Dysfunctions Events. The statistical data was significantly favorable to covered SEMS group at incidence of adverse events ( 8 trials reported the data, OR: $0.74,95 \% \mathrm{CI}: 0.57$ to $0.97, P=0.03$ ) (Figure 4 ), and there is a lower trend toward dysfunction events ( 8 trials reported the data, OR: $0.75,95 \%$ CI: 0.56 to $1.00, P=0.05$ ) (Figure 5). However, there is no significant difference in complications (OR: $0.87,95 \%$ CI: 0.58 to $1.30, P=0.50$ ) (Figure 6). According to the statistical data, the incidence of adverse events is lower in the covered SEMS, while there is no difference in complications.

3.5. Publication Bias. The publication bias of included studies was evaluated by funnel plots. As is shown in Figure 8, the funnel plots were almost symmetric. Hence, there was no evidence for significant publication bias in our meta-analysis.

\section{Discussion}

This meta-analysis shows that the covered SEMS group had lower incidence of adverse events (dysfunctions and complications) than the uncovered SEMS treatment group for unresectable distal malignant biliary obstructions. The main cause may be the higher reobstruction rates, which result from the tumor ingrowth and overgrowth after uncovered SEMS replacement. Although migration can contribute to the covered SEMS reobstruction or dysfunctions, the incidence 


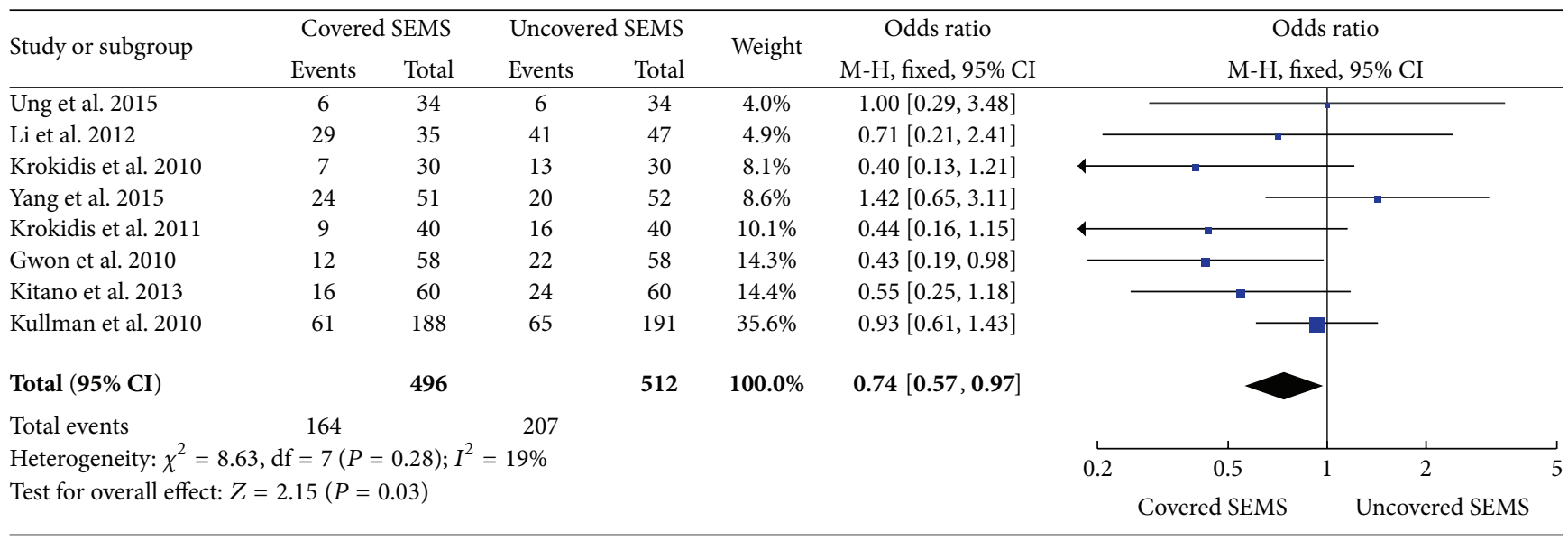

FIGURE 4: Forest plot of studies evaluating odds ratios of adverse events.

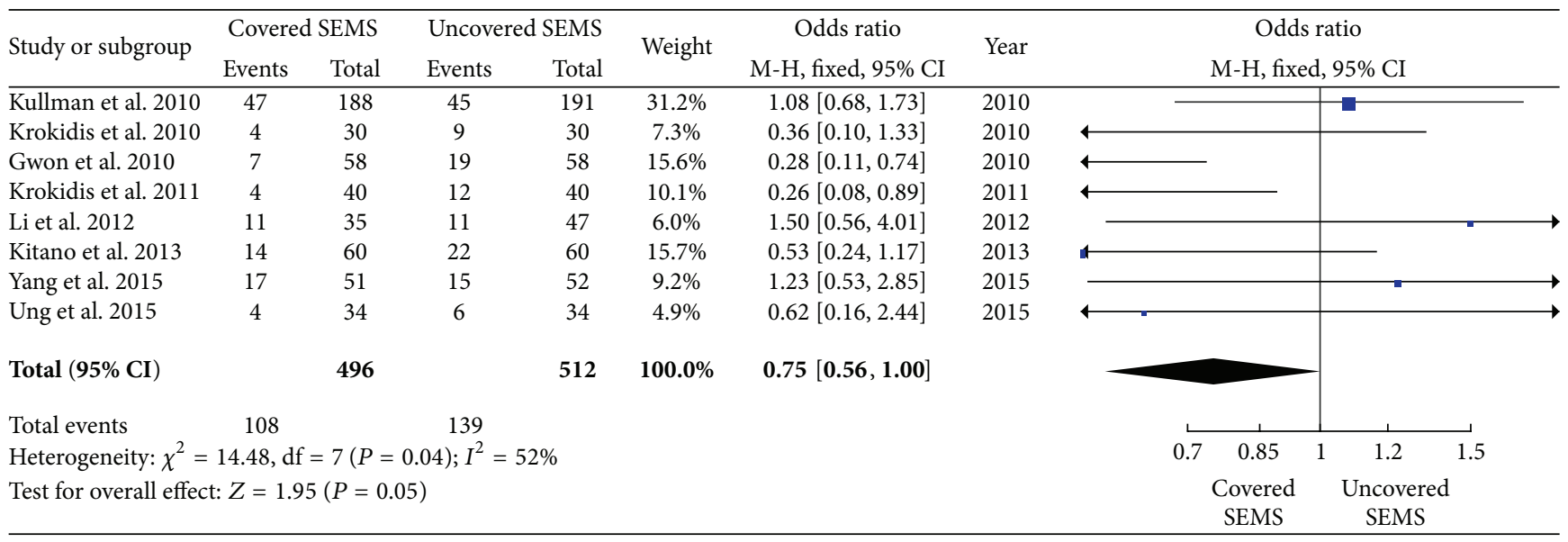

FIGURE 5: Forest plot of studies evaluating odds ratios of dysfunctions events.

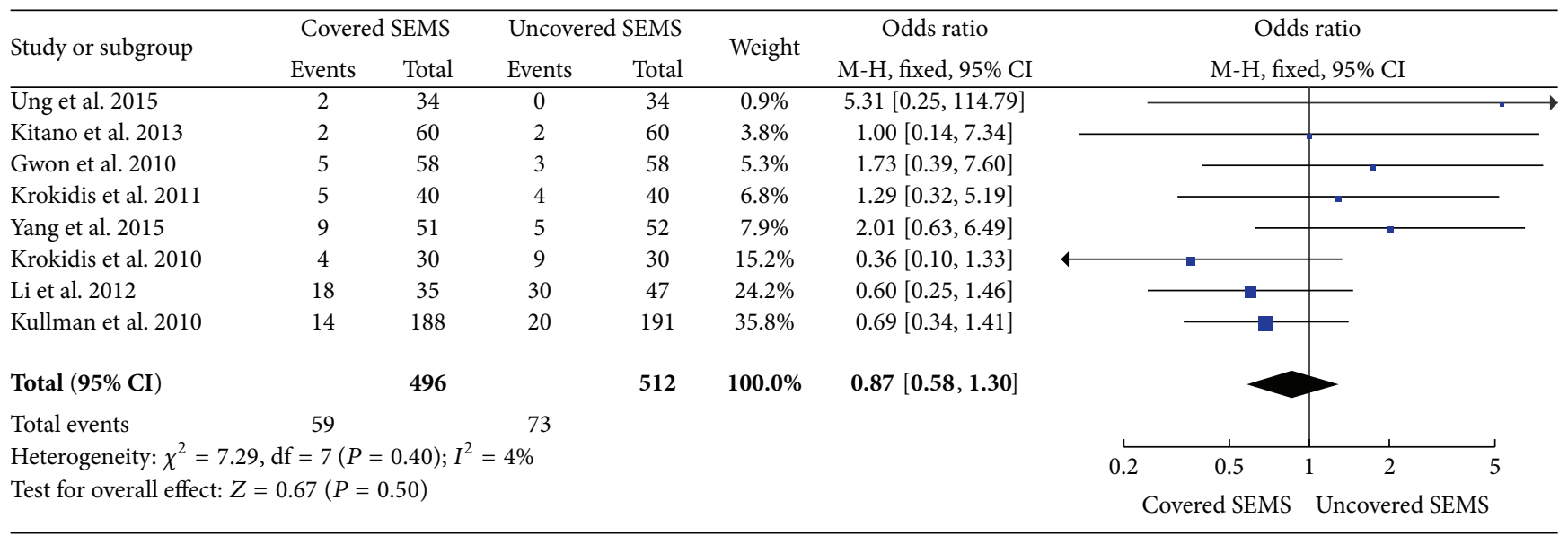

FIGURE 6: Forest plot of studies evaluating odds ratios of complications. 


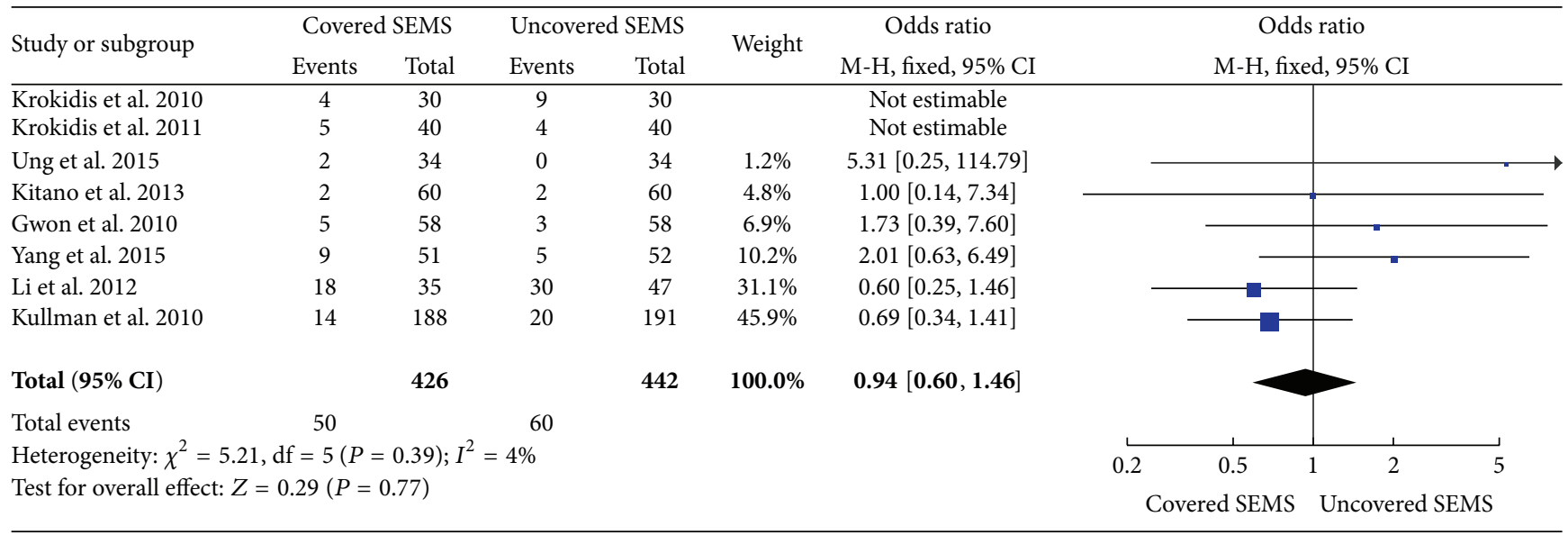

FIGURE 7: Forest plot of studies evaluating odds ratios of complications with endoscopic stent placement.

may be lower than tumor ingrowth or overgrowth rate after uncovered SEMS replacement. Moreover, although the incidence of complications was not significantly different between covered and uncovered SEMS, a trend was shown in the rates of dysfunctions (OR: $0.75,95 \% \mathrm{CI}: 0.56$ to 1.00 , $P=0.05)$. This may explain better outcomes using covered SEMS.

Our results also suggest that covered SEMS should not provide a significant prolongation in cumulative stent patency when compared with uncovered SEMS. Although stent dysfunction had a trend towards being lower in covered SEMS at a given time, the stent patency was influenced by various factors, such as structural properties of SEMSs and presence of covering materials [21]. However, in the 8 trials, the follow-up end point was either last follow-up or patient death, and there was no difference in patient survival.

Acute pancreatitis and acute cholecystitis are the potential of complications, when the covered SEMS is placed over the cystic or pancreatic duct orifice. Although not proven in clinical studies, it has been claimed that covered SEMS might increase the prevalence of pancreatitis or cholecystitis by blocking the cystic duct orifice or pancreatic duct orifice. However, in our studies, we cannot come to the conclusion that the rate of complications was significant. It is probably due to the high portion of pancreatic cancer patients in the two groups. Additionally, this observation is commonly confounded by a low event rate [22]. What is more, such measures or precision may not be used widely in clinical practice in some hospitals. Therefore, it must be noted that measures were taken to reduce the rate of complications, either by using covered SEMSs with transmural drainage holes $[8,9]$ or by ensuring that the stent covering was placed below the level of the cystic or pancreatic duct.

In patients with unresectable malignant distal biliary obstruction, palliation may be offered with stent placement (either endoscopic or percutaneous). Endoscopic biliary stent placement seems to be associated with lower complication rate and better quality of life [23]. Therefore, endoscopic biliary stent placement is considered the gold standard, in order to improve success rates and patient outcomes. However, some studies $[8,9]$ we selected in this meta-analysis included percutaneous biliary stent placement, although ERCP was the initial approach, and PTC was performed when ERCP was not feasible. We cannot know how many participate in percutaneous biliary stent placement from the original article, but we get the same result in evaluating odds ratios of complications with endoscopic stent placement (OR: 0.94, 95\% CI: 0.60 to $1.46, P=0.39$ ) (Figure 7).

4.1. Limitations and Strengths. Limitations of our analysis include the use of various covered SEMSs; ePTFE/FEP was used in two studies, whereas other materials (polyurethane, polycarbonate-polyurethane, and Permalume) were used in the remaining studies. Therefore, we assumed there were no differences in different types of covered SEMSs, like some published trials $[4,16,24,25]$.

The strength of this meta-analysis comes from the high methodological quality of each individual study as well as data homogeneity for most outcomes, including the primary outcome of stent patency, patient survival, and incidence of adverse events (dysfunctions and complications).

\section{Conclusions}

Covered SEMS group had lower incidence of adverse events (dysfunctions and complications) than the uncovered SEMS group. There is no significant difference in dysfunctions, but covered SEMS trends to be better, and there is no difference in stent patency, patient survival, and complications. Compared with uncovered SEMS, covered SEMS is recommended for treating patients who are diagnosed as having unresectable distal malignant biliary obstructions.

\section{Conflict of Interests}

The authors declare that there is no conflict of interests regarding the publication of this paper. 


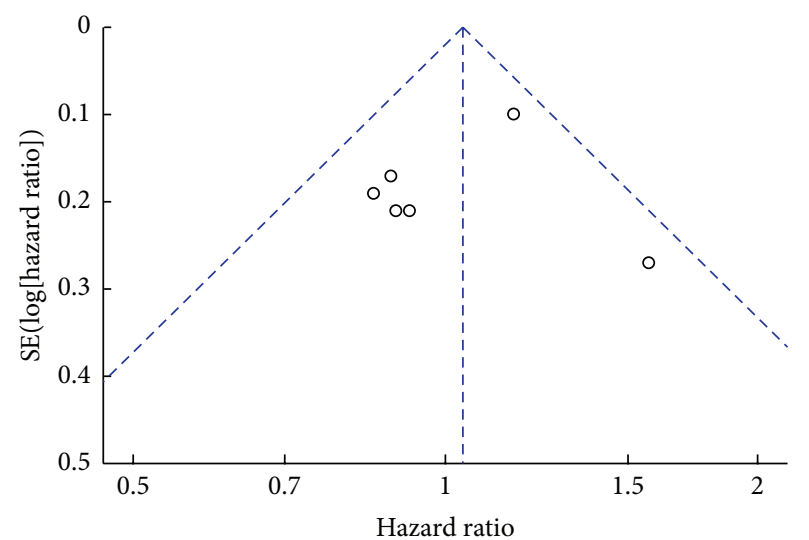

(a)

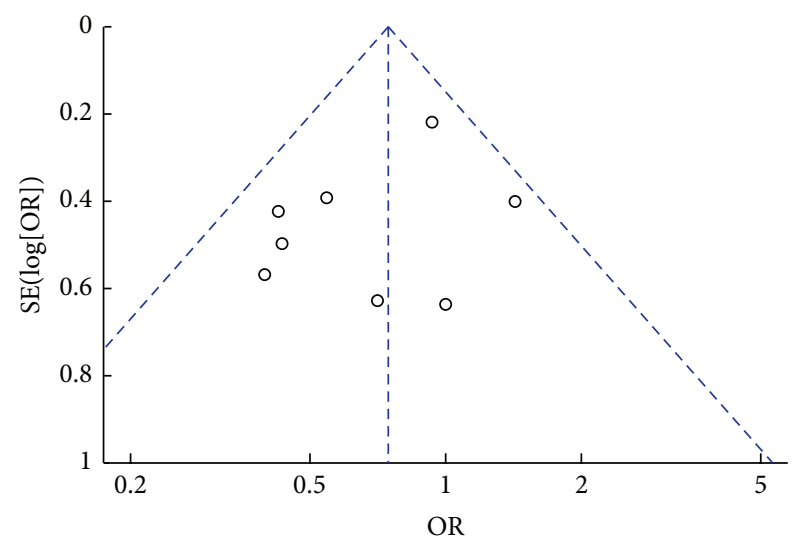

(c)

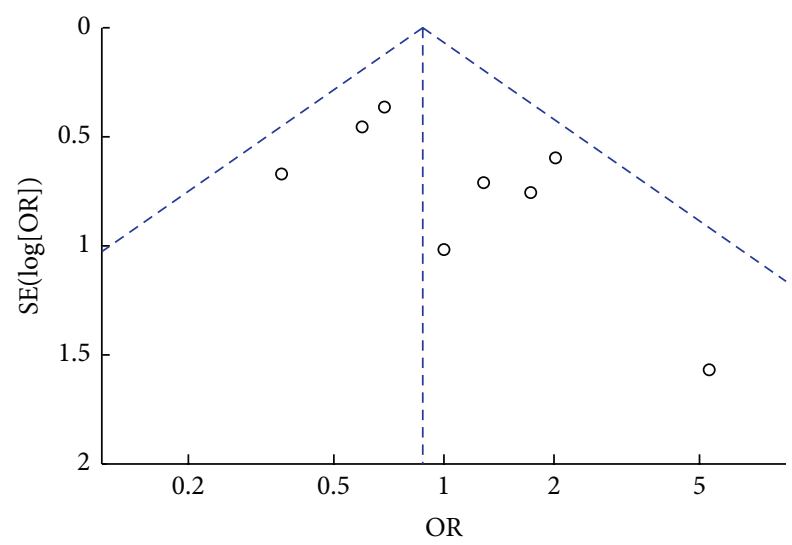

(e)

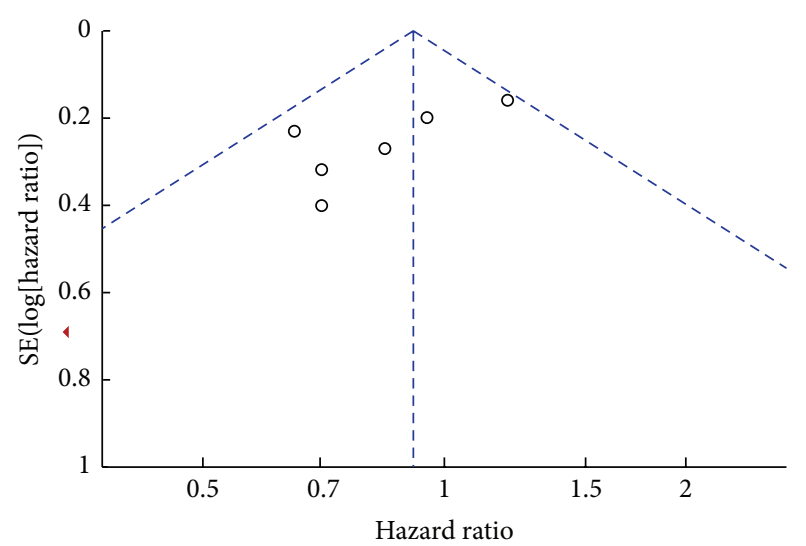

(b)

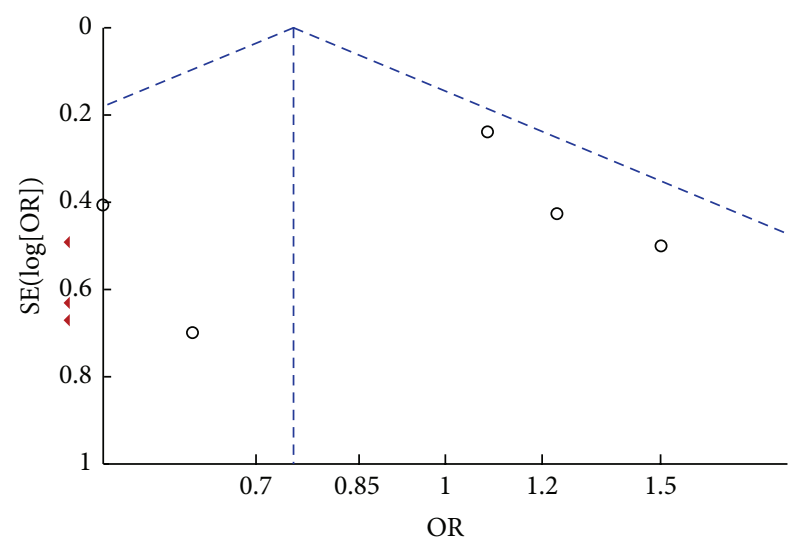

(d)

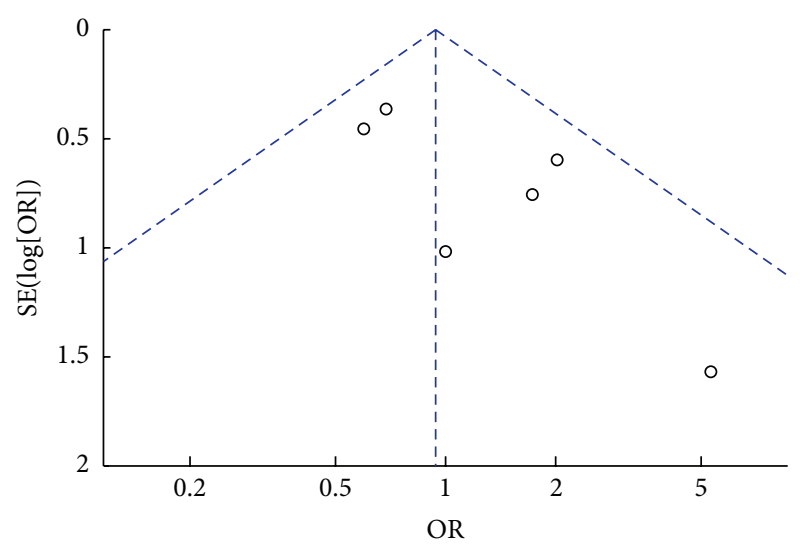

(f)

FIGURE 8: Funnel plot of studies included in the six meta-analyses: (a) patient survival, (b) stent patency, (c) adverse events, (d) dysfunctions events, (e) complications, and (f) complications with endoscopic stent placement.

\section{Authors' Contribution}

Ming-Yu Chen and Xiu-Jun Cai wrote the paper and performed the research, Xiu-Jun Cai and Jia-Wei Lin designed the study, He-Pan Zhu and Bin Zhang collected the data, Guang-Yi Jiang and Pei-Jian Yan performed the literature search and retrieved the data, and Xiu-Jun Cai analyzed the data.

\section{References}

[1] N. Soehendra and V. Reynders-Frederix, "Palliative bile duct drainage-a new endoscopic method of introducing a transpapillary drain," Endoscopy, vol. 12, no. 1, pp. 8-11, 1980.

[2] E. Kullman, F. Frozanpor, C. Sderlund et al., "Covered versus uncovered self-expandable nitinol stents in the palliative treatment of malignant distal biliary obstruction: results from 
a randomized, multicenter study," Gastrointestinal Endoscopy, vol. 72, no. 5, pp. 915-923, 2010.

[3] T. Itoi, K. Kasuya, Y. Abe, and H. Isayama, "Endoscopic placement of a new short-term biodegradable pancreatic and biliary stent in an animal model: a preliminary feasibility study (with videos)," Journal of Hepato-Biliary-Pancreatic Sciences, vol. 18, no. 3, pp. 463-467, 2011.

[4] H. Isayama, Y. Komatsu, T. Tsujino et al., "A prospective randomised study of 'covered' versus 'uncovered' diamond stents for the management of distal malignant biliary obstruction," Gut, vol. 53, no. 5, pp. 729-734, 2004.

[5] J. J. Telford, D. L. Carr-Locke, T. H. Baron et al., "A randomized trial comparing uncovered and partially covered selfexpandable metal stents in the palliation of distal malignant biliary obstruction," Gastrointestinal Endoscopy, vol. 72, no. 5, pp. 907-914, 2010.

[6] M. A. Almadi, A. N. Barkun, and M. Martel, "No benefit of covered vs uncovered self-expandable metal stents in patients with malignant distal biliary obstruction: a meta-analysis," Clinical Gastroenterology and Hepatology, vol. 11, no. 1, pp. 2737, 2013.

[7] D. I. Gwon, G.-Y. Ko, J. H. Kim et al., "A comparative analysis of PTFE-covered and uncovered stents for palliative treatment of malignant extrahepatic biliary obstruction," American Journal of Roentgenology, vol. 195, no. 6, pp. W463-W469, 2010.

[8] M. Krokidis, F. Fanelli, G. Orgera, M. Bezzi, R. Passariello, and A. Hatzidakis, "Percutaneous treatment of malignant jaundice due to extrahepatic cholangiocarcinoma: covered Viabil stent versus uncovered Wallstents," CardioVascular and Interventional Radiology, vol. 33, no. 1, pp. 97-106, 2010.

[9] M. Krokidis, F. Fanelli, G. Orgera et al., "Percutaneous palliation of pancreatic head cancer: randomized comparison of ePTFE/FEP-covered versus uncovered nitinol biliary stents," CardioVascular and Interventional Radiology, vol. 34, no. 2, pp. 352-361, 2011.

[10] F. Li, F. Wang, X. Yang et al., "Covered stents versus uncovered stents for the palliation of malignant extrahepatic biliary obstruction caused by direct tumor invasion: a cohort comparative study," Medical Oncology, vol. 29, no. 4, pp. 2762-2770, 2012.

[11] M. Kitano, Y. Yamashita, K. Tanaka et al., "Covered selfexpandable metal stents with an anti-migration system improve patency duration without increased complications compared with uncovered stents for distal biliary obstruction caused by pancreatic carcinoma: a randomized multicenter trial," American Journal of Gastroenterology, vol. 108, no. 11, pp. 1713-1722, 2013.

[12] K.-A. Ung, P.-O. Stotzer, Å. Nilsson, M.-L. Gustavsson, and E. Johnsson, "Covered and uncovered self-expandable metallic Hanarostents are equally efficacious in the drainage of extrahepatic malignant strictures. Results of a double-blind randomized study," Scandinavian Journal of Gastroenterology, vol. 48, no. 4, pp. 459-465, 2015.

[13] M. J. Yang, J. H. Kim, B. M. Yoo et al., "Partially covered versus uncovered self-expandable nitinol stents with anti-migration properties for the palliation of malignant distal biliary obstruction: a randomized controlled trial," Scandinavian Journal of Gastroenterology, vol. 50, no. 12, pp. 1490-1499, 2015.

[14] J. F. Tierney, L. A. Stewart, D. Ghersi, S. Burdett, and M. R. Sydes, "Practical methods for incorporating summary time-toevent data into meta-analysis," Trials, vol. 8, article 16, 2007.

[15] M. K. B. Parmar, V. Torri, and L. Stewart, "Extracting summary statistics to perform meta-analyses of the published literature for survival endpoints," Statistics in Medicine, vol. 17, no. 24, pp. 2815-2834, 1998.

[16] P. Katsinelos, J. Kountouras, G. Paroutoglou et al., "Uncovered Hanaro versus Luminex metal stents for palliation of malignant biliary strictures," Journal of Clinical Gastroenterology, vol. 42, no. 5, pp. 539-545, 2008.

[17] T. J. Song, S. S. Lee, S. C. Yun et al., "Paclitaxel-eluting covered metal stents versus covered metal stents for distal malignant biliary obstruction: a prospective comparative pilot study," Gastrointestinal Endoscopy, vol. 73, no. 4, pp. 727-733, 2011.

[18] H. Isayama, Y. Nakai, H. Kogure, N. Yamamoto, and K. Koike, "Biliary self-expandable metallic stent for unresectable malignant distal biliary obstruction: which is better: covered or uncovered?" Digestive Endoscopy, vol. 25, no. 2, pp. 71-74, 2013.

[19] J. H. Lee, S. G. Krishna, A. Singh et al., "Comparison of the utility of covered metal stents versus uncovered metal stents in the management of malignant biliary strictures in 749 patients," Gastrointestinal Endoscopy, vol. 78, no. 2, pp. 312-324, 2013.

[20] P. L. Moses, K. M. Alnaamani, A. N. Barkun et al., "Randomized trial in malignant biliary obstruction: plastic vs partially covered metal stents," World Journal of Gastroenterology, vol. 19, no. 46, pp. 8638-8646, 2013.

[21] W. J. Yoon, J. K. Lee, K. H. Lee et al., "A comparison of covered and uncovered Wallstents for the management of distal malignant biliary obstruction," Gastrointestinal Endoscopy, vol. 63, no. 7, pp. 996-1000, 2006.

[22] A. Saleem, C. L. Leggett, M. H. Murad, and T. H. Baron, "Metaanalysis of randomized trials comparing the patency of covered and uncovered self-expandable metal stents for palliation of distal malignant bile duct obstruction," Gastrointestinal Endoscopy, vol. 74, no. 2, pp. 321-327.e3, 2011.

[23] T. H. Lee, S. J. Lee, J. H. Moon, and S.-H. Park, “Technical tips and issues of biliary stenting, focusing on malignant hilar obstruction," Minerva Gastroenterologica e Dietologica, vol. 60, no. 2, pp. 135-149, 2014.

[24] J. M. Dumonceau, M. Cremer, J. Auroux, M. Delhaye, and J. Devière, "A comparison of ultraflex diamond stents and wallstents for palliation of distal malignant biliary strictures," American Journal of Gastroenterology, vol. 95, no. 3, pp. 670676, 2000.

[25] R. J. Shah, D. A. Howell, D. J. Desilets et al., "Multicenter randomized trial of the spiral Z-stent compared with the Wallstent for malignant biliary obstruction," Gastrointestinal Endoscopy, vol. 57, no. 7, pp. 830-836, 2003. 


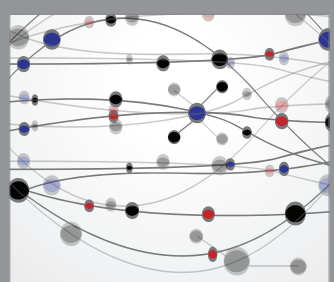

The Scientific World Journal
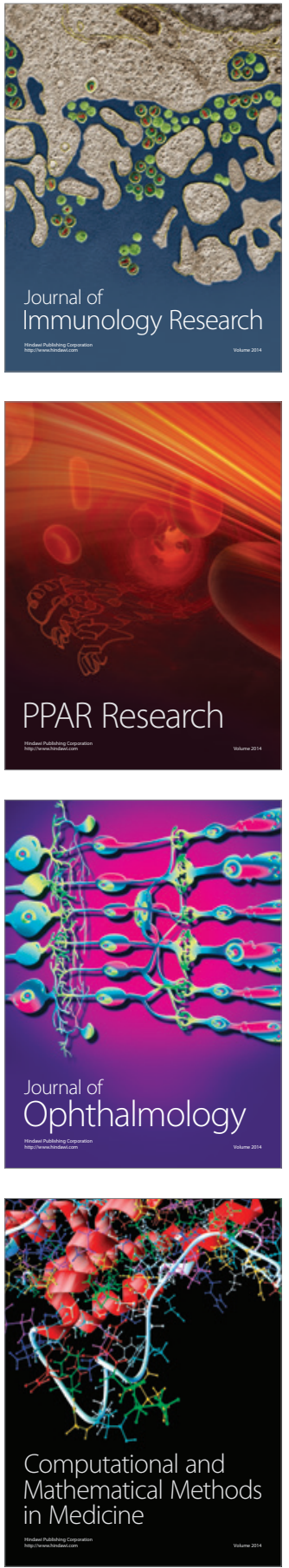

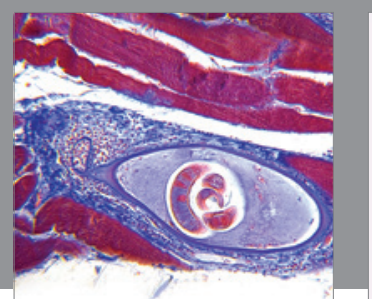

Gastroenterology Research and Practice

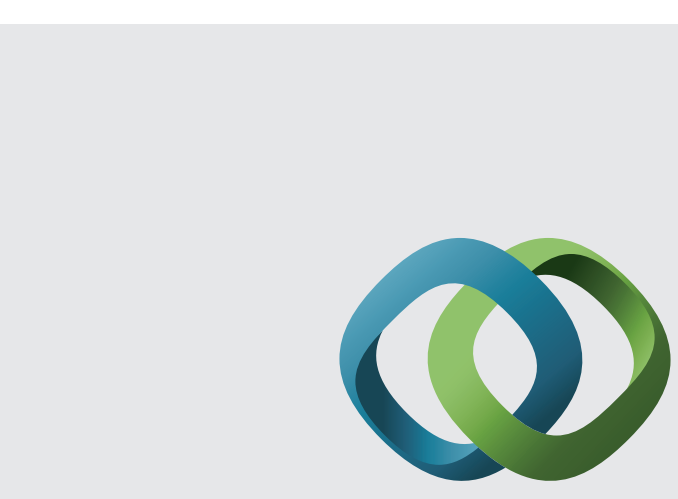

\section{Hindawi}

Submit your manuscripts at

http://www.hindawi.com
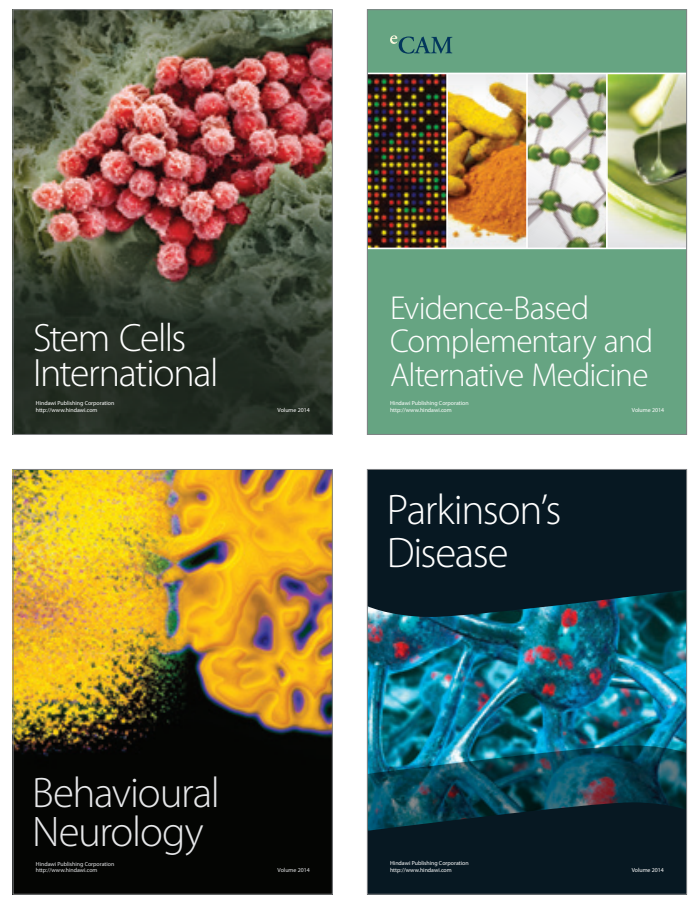
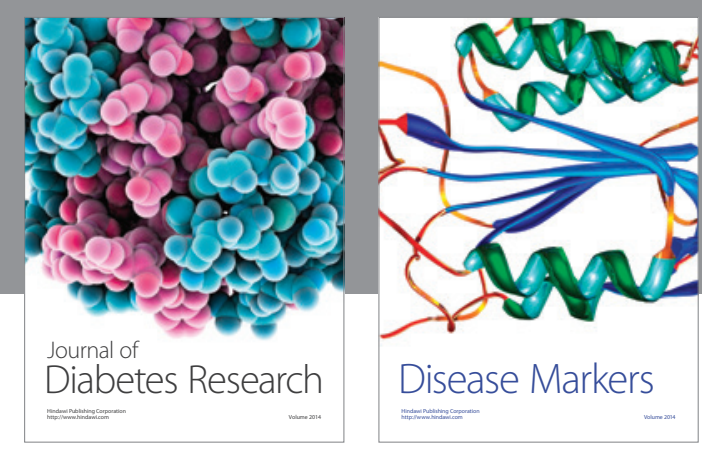

Disease Markers
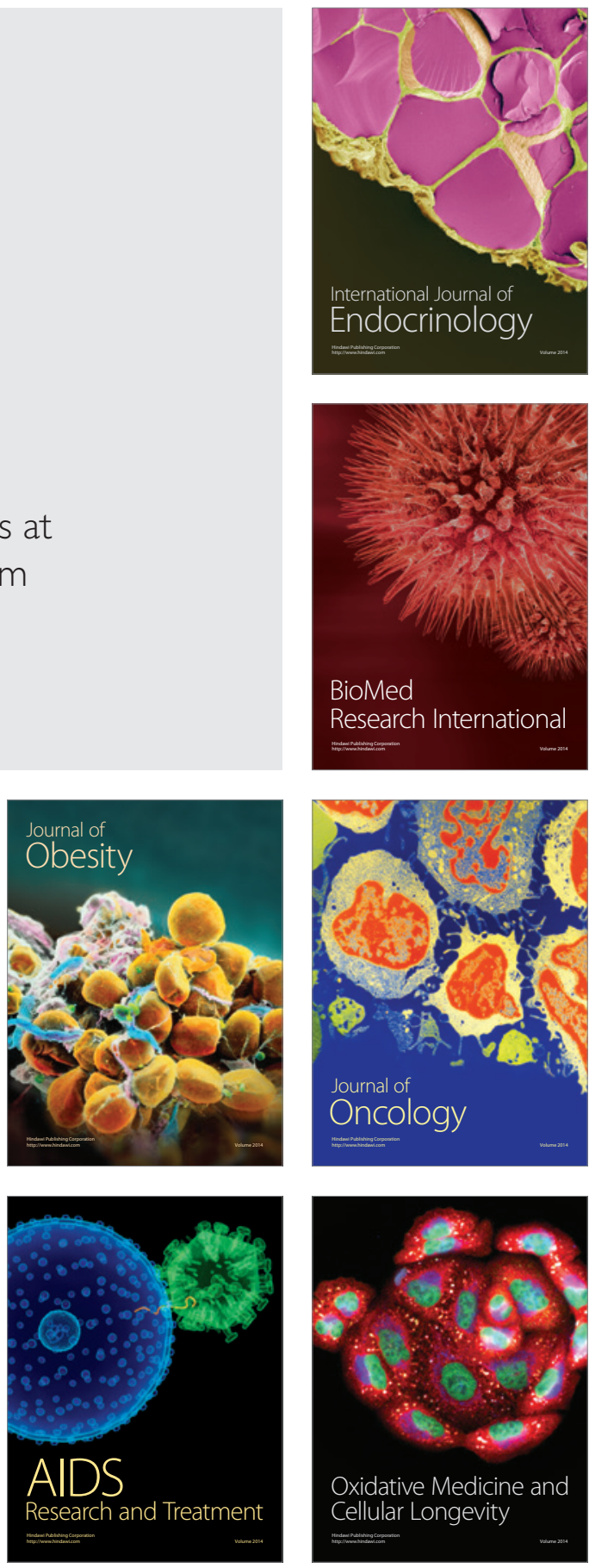